\title{
SEAPAL quality control analysis of rheumatoid factor measurement in 29 diagnostic laboratories
}

\author{
P J ROBERTS-THOMSON, ${ }^{1}$ T LANGHANS, ${ }^{1}$ R McEVOY, ${ }^{1}$ \\ P N HOLLINGSWORTH, ${ }^{2}$ E BONIFACIO, ${ }^{2} R$ L DAWKINS, ${ }^{2}$ AND \\ J B R A DLE Y
}

From the ${ }^{\prime}$ Department of Clinical Immunology, Flinders Medical Centre, Bedford Park, South Australia; and the ${ }^{2}$ Department of Clinical Immunology, The Queen Elizabeth II Medical Centre, Nedlands, Western Australia

SUMmaRy A programme for rheumatoid factor (RF) measurement was sponsored by the South East Asian Pacific League against Rheumatism (SEAPAL) and the Clinical Immunology Group (CIG) of the Australian Society for Immunology. Measurements in 29 laboratories in 12 countries showed: $(a)$ a large variety of methods were being used to measure RF, most using semiquantitative agglutination techniques; $(b)$ some laboratories have invalid techniques; $(c)$ the quoted upper normal limit varied considerably between assays, and false positives were identified in three laboratories; $(d)$ a wide range of intralaboratory precision within and between assays; and $(e)$ a reduction in discordance between laboratories by the inclusion of local standards. Before international standardisation of RF measurement can be achieved attention to many of these problems is required. In particular we recommend the wider use of reference standards and the regular participation in quality control programmes in order to monitor performance.

The standardisation of the measurement of RF has proved surprisingly difficult. ${ }^{1}$ If standardisation could be achieved it would allow for greater uniformity in applying seropositivity as one diagnostic criterion for rheumatoid arthritis. Furthermore, and perhaps more importantly, it would then be possible to examine critically the value of quantitative measurement of RF as a means of predicting the outcome of patients and in monitoring the response to disease modifying drugs such as gold. ${ }^{2}$ As part of the wider aims of SEAPAL and CIG, 29 laboratories in South East Asia and other countries have participated in a quality control programme of autoantibodies, including RF. The aims of the RF study have been to assess $(a)$ the methods currently in use for measuring RF; $(b)$ the validity of the methods; $(c)$ intra- and interlaboratory reproducibility; and $(d)$ the degree of concordance between laboratories and whether the inclusion of standards can improve this concordance.

Accepted for publication 31 October 1986.

Correspondence to $\mathrm{Dr} \mathrm{P} \mathrm{J}$ Roberts-Thomson, Department of Clinical Immunology, Flinders Medical Centre, Bedford Park, South Australia 5042.

\section{Materials and methods}

QUALITY CONTROL EXCHANGE

The 29 laboratories who took part in the exchange were requested to supply details of their methods and to designate the cut off value which they considered differentiated normal from abnormally raised values (i.e., upper normal limit). Test serum samples were distributed to the laboratories on two occasions approximately three months apart. There were four test samples on the first occasion, A to D. $\mathrm{A}, \mathrm{B}$, and $\mathrm{C}$ consisted of standards containing RF in increasing quantities (vide infra) for use in assessing the validity of the method and to allow the construction of a standard curve, and sample $D$ was an 'unknown' seropositive specimen. On the second occasion a further four samples E, F, G, and $\mathrm{H}$ were circulated. Samples $\mathrm{E}$ and $\mathrm{H}$ were duplicates of $\mathrm{A}$ to determine within assay and between assay reproducibility; sample $G$ was a duplicate of $D$ to compare values between laboratories and to determine between assay reproducibility; and sample $F$ was without detectable RF to detect false positives.

PREPARATION OF STANDARDS A, B, AND C Five rheumatoid arthritis sera containing high levels 

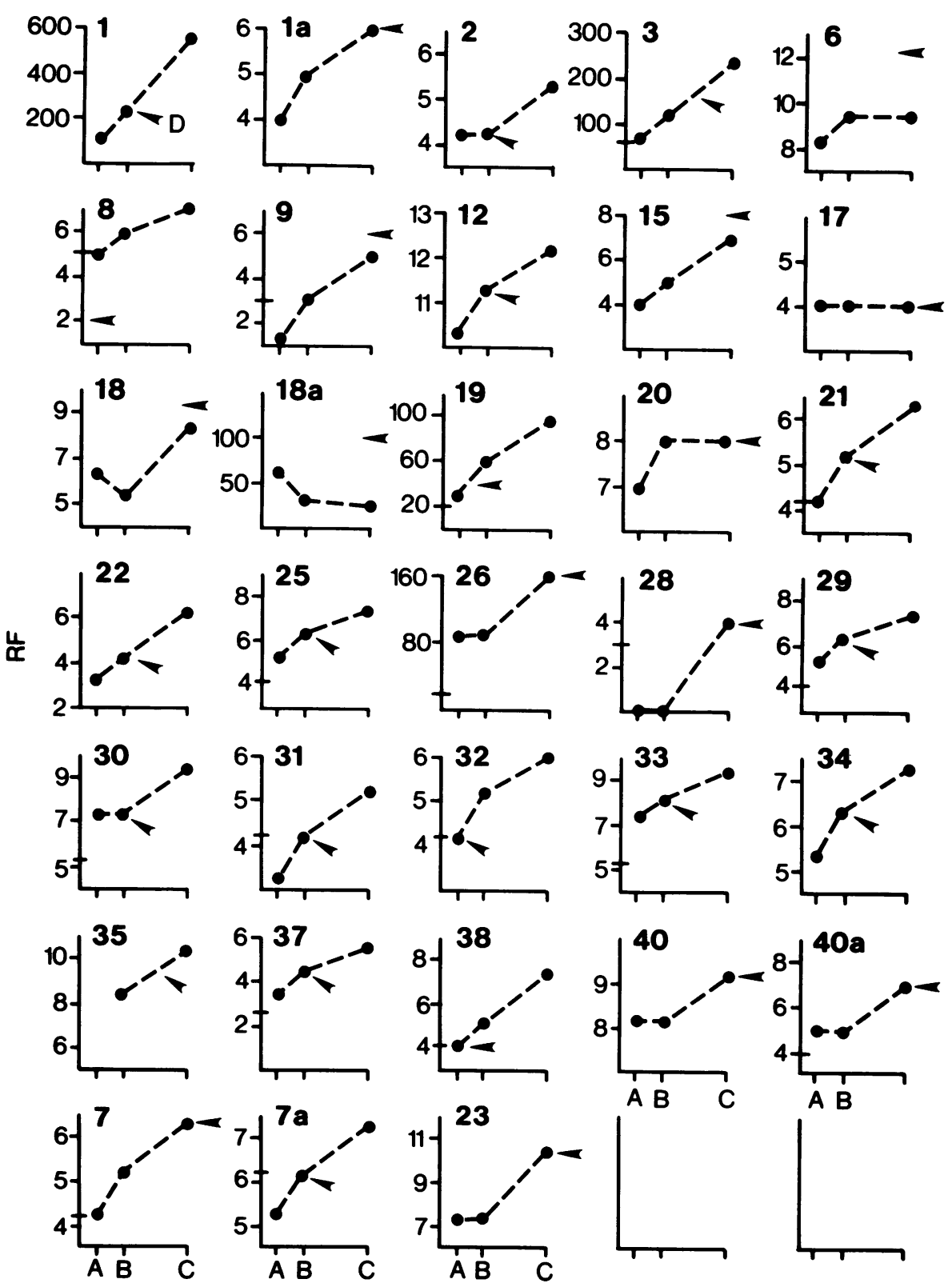

A B
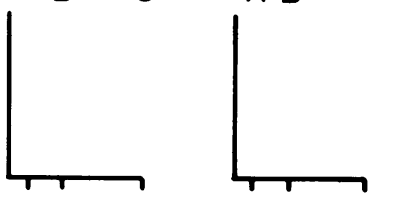

LOCAL STANDARDS A,B \& C

Fig. 1 Graphically displayed laboratory results for local standards $A, B$, and $C$ (to demonstrate the validity of the metho $\overrightarrow{0}$ and for sample $D$ ('unknown' seropositive specimen, whose value is indicated by the arrow in each panel). The laboratorie are numbered 1 to 40 (with the 11 omitted numbers representing laboratories who did not return their results). The quoted upper normal limit for each assay (when known) is represented by a bar on the y ordinate. The reciprocal of the $\log _{2}$ of the RF titre or absolute level of RF is also indicated on this ordinate. 
of $\mathrm{RF}$ (>9999 IU/ml) were pooled and added to an equal volume of $6 \%$ polyethylene glycol (PEG) 6000 in phophate buffered saline (PBS) at $\mathrm{pH} 7 \cdot 4$. After standing at $4^{\circ} \mathrm{C}$ overnight the solution was centrifuged at $1000 \mathrm{~g}$ for $20 \mathrm{~min}$ and the precipitate collected and washed in cold 3\% PEG 6000 and recentrifuged. The collected precipitate was then dissolved in a small volume (3-4 ml) of PBS and empirical small volumes added to pooled normal serum (final dilutions 1:160, 1:100, 1:40) to prepare three standards containing increasing quantities of RF. By rate nephelometry (Beckmans ICS II) standard A contained $81 \mathrm{IU} / \mathrm{ml}$ of $\mathrm{RF}$ (mean of three readings), standard B $142 \mathrm{IU} / \mathrm{ml}$, and standard C $290 \mathrm{IU} / \mathrm{ml}$. Sample D was derived from a donor whose serum contained $163 \mathrm{IU} / \mathrm{ml}$ of RF. Sample F contained no detectable RF, and the pooled normal serum used as diluent for standards $\mathrm{A}, \mathrm{B}$, and $\mathrm{C}$ was derived from a single donor. Aliquots $(500 \mu \mathrm{l})$ of all samples were stored at $-20^{\circ} \mathrm{C}$ until delivered. Sodium azide was added to all samples as a preservative. The maximum variation of $R F$ measurement noted in any of the positive standards after standing for nine days at room temperature was $4 \cdot 6 \%$. The samples were not checked for hepatitis B antigen or acquired immune deficiency syndrome related virus, and each participating laboratory was warned of potential infectivity.

\section{Results}

Twenty nine laboratories took part in the exchange (Table 1). A number of laboratories used more than one method to measure RF, the most popular being a commercially derived latex agglutination technique (Table 2). In addition, a wide variety of other methods were noted, and only one laboratory was using a locally developed assay (ELISA).

Most laboratories were able to demonstrate the validity of their method as reflected by increasing levels of $R F$ when measuring samples $A$ to $C$, but

Table 1 Origin of participating laboratories

\begin{tabular}{lc}
\hline Country & Number \\
\hline Australia & 13 \\
China & 1 \\
Hong Kong & 1 \\
India & 2 \\
Japan & 2 \\
Malaysia & 2 \\
New Zealand & 2 \\
Singapore & 1 \\
Thailand & 1 \\
United Kingdom & 2 \\
United States & 2 \\
\hline
\end{tabular}

Table 2 Different methods used to measure RF

\begin{tabular}{lcc}
\hline Method & $\begin{array}{l}\text { No of } \\
\text { laboratories }\end{array}$ & $\begin{array}{l}\text { No of } \\
\text { variations } \\
\text { of methods }\end{array}$ \\
\hline Sheep cell agglutination & 11 & 3 \\
Latex agglutination & 23 & 10 \\
Nephelometry & 2 & 1 \\
ELISA* & 1 & 1 \\
\hline
\end{tabular}

${ }^{*}$ ELISA $=$ enzyme linked immunosorbent assay.

there were two notable exceptions-laboratories Nos 17 and 18 (Fig. 1). It is also apparent from this figure that the quoted upper normal limit of RF varied considerably between laboratories, and a wide variation was observed between laboratories when this value was compared with sample $A$ which was designed to contain low but discriminating levels of RF. In some laboratories (e.g., Nos 26 and 30) the quoted upper normal limit was well below that obtained for this sample, while in others (e.g., 9 and 28) it was well above this level. Furthermore, three laboratories (Nos 17, 20, and 40) detected RF in the seronegative sample $F$ (i.e., false positive). Using samples $\mathrm{A}, \mathrm{B}$, and $\mathrm{C}$ as internal standards to construct a standard curve, most laboratories (21/29) correctly identified specimen D as falling at or between standards B and C (Fig. 1). The mean difference in titre $\left(\log _{2}\right)$ (SD) for within assay duplicates (samples $E$ and $H$ ) was $0.29(0.83)$, whereas for between assay duplicates (samples D and $\mathrm{G})$ it was $0.77(0.66)$.

\section{Discussion}

This SEAPAL quality control analysis of RF measurement in 29 diagnostic laboratories in 12 countries has confirmed the results of previous similar studies showing wide variation in RF levels between laboratories even when similar methods are compared. ${ }^{34}$ For example, in the present study a 30fold difference (from $1 / 40$ to $1 / 1280$ ) was found on the same seropositive serum (sample D) using the latex agglutination technique. This gross discordance was greatly improved, however, by the inclusion of the local reference standard A, B, and $\mathrm{C}$, suggesting that interlaboratory variation could be markedly diminished by this approach, and this finding is in accord with other studies. ${ }^{5} 6$ It was disappointing to note that even though an international reference preparation of $\mathrm{RF}$ has been available for 15 years $^{7}$ and has been strongly recommended as improving concordance between laboratories, only eight laboratories were expressing their results in international units. 
Most laboratories were able to demonstrate the validity of their methods as reflected by linearity with increasing quantities of RF (standard $A$ to $C$ ) and had acceptable within assay and between assay precision. There were some notable exceptions, however, and it is hoped that one of the benefits of this study will be to alert such laboratories. The quoted upper normal limit of each laboratory's technique varied considerably between laboratories. Certain laboratories were identified where the upper limit appeared inappropriate. The assessment of sensitivity and specificity should ideally be performed in each laboratory using locally derived test and control specimens.

Agglutination techniques are only semiquantitative and suffer in that absolute values of RF have to change by $50 \%$ before there is a change of titre (using the double dilution technique). We would recommend that large diagnostic laboratories consider using more accurate methods to measure RF, particularly if they use RF to monitor the response of patients to disease modifying medications. Such methods currently available include nephelometry ${ }^{x}$ (used by two laboratories-Nos 1 and 3 in the present study) and ELISA" (used by one laboratory-No 19). These methods are sensitive, quantitative, and precise, and the nephelometric technique is rapid, results being available within minutes. The disadvantages of these methods in comparison with the simple latex agglutination technique is the cost of the initial outlay for the nephelometer or spectrophotometer (ELISA). When economic restraints are necessary simple latex agglutination methods calibrated in international units seem appropriate.

We would strongly recommend that laboratories regularly participate in quality control programmes involving RF measurement. We have unpublished data from an earlier quality control programme involving complement measurements which indicate that such regular participation encourages enhanced precision in these measurements by alerting errare laboratories of their poor performance and leadin to subsequent corrections or modifications.

In conclusion, we noted considerable variations the methods used to measure RF in this quali control programme, identified certain laboratories with invalid techniques, and identified much inte 8 laboratory variance, which was reduced by the inclusion of standards. We would recommend that these identified deficiencies are corrected and wouf encourage the use of reference standards to allow results to be expressed in international units. Fu@ thermore, we would encourage all laboratorisg that measure RF to participate regularly in qualite control programmes in order to monitor the f performance.

We thank Mrs M Brown for typing the manuscript, and tof participants who were involved in this study.

\section{References}

1 Taylor R N. Measurement of variation and significance $\vec{\varphi}$ serologic tests. Ann NY Acad Sci 1983: 420: 13-21.

2 Pope R M. Lessard J. Nunnery E. Differential effects is therapeutic regimens on specific classes of rheumatoid factor. Ann Rheum Dis 1986; 45: 183-9.

3 Bozsoky S. Problem of standardisation in rheumatoid-arthri scrology. Ann Rheum Dis 1963; 6: 641-9.

4 Klein F, Valkenburg H A, Cats A. On standardization of ti latex fixation test. Bull Rheum Dis 1975; 26: 866-8.

5 Fulford K M. Taylor R N. Przybyszewski V A. Refereng preparation to standardize results of scrological tests fog rhcumatoid factor. J Clin Microbiol 1978: 7: 434-41.

6 Taylor R N. Fulford K M. Jones W L. Reduction of variation results of rheumatoid factor tests by use of a serum reference preparation. J Clin Microbiol 1977; 5: 42-5.

7 Anderson S G. Bentzon M W. Hooba V. Krayo P. Intco national reference preparations of rheumatoid arthritis seruro Bull WHO 1970): 42: 311-18.

8 Roberts-Thomson P J. McEvoy R. Langhans T, Bradley Routine quantification of rheumatoid factor by rate neph $\Phi$ ometry. Ann Rheum Dis 1985; 44: 379-83.

9 Therewis W. Highton J, Palmer D O. Assessment of a simpte ELISA assay for rheumatoid factor in clinical practice. Prö Unw Otago Med Sch 1982; 60: 83-4. 